\title{
Resultados del tratamiento de la estenosis aórtica en niños de un Hospital Universitario
}

\author{
Results of aortic stenosis treatment in children at a \\ University Hospital
}

\author{
Dong Chin Suh", Milagros Varga-Peña ${ }^{1}$, Junior Greco ${ }^{1}$, Lina Villagra ${ }^{1}$, Patricia Pereira Dick ${ }^{1}$, \\ Amalio Benítez ${ }^{1}$, Patricia Egusquiza', Jorge Jarolín ${ }^{1}$
}

\section{RESUMEN}

Introducción: La estenosis aórtica es relativamente frecuente en niños. El objetivo de este trabajo fue de caracterizar los resultados y la evolución de esta patología luego del manejo quirúrgico y percutáneo. Material y Métodos: Estudio retrospectivo de pacientes de cero a 19 años, tratados de estenosis aórtica subvalvular, valvular y supravalvular, en el Hospital de Clínicas, entre 1998 y 2019. Fueron excluidos los casos asociados a otras lesiones congénitas. Resultados: Fueron tratados 20 pacientes, edad de 3 días a 17 años, predominio masculino (80\%). La estenosis fue valvular en $65 \%$ de los casos, subvalvular $20 \%$, supravalvular 5\% y múltiple en $10 \%$. El $61 \%$ de los valvulares recibió tratamiento percutáneo, y los demás valvuloplastia quirúrgica. En el seguimiento, la tasa libre de reintervención fue de $47 \%$ a 10 años; el $50 \%$ de ellos tiene insuficiencia valvular aórtica moderada a severa. De los 13 casos de estenosis valvular, tratados percutánea o quirúrgicamente, 4 están aguardando recambio valvular. De 4 pacientes con estenosis subvalvular, 3 tienen insuficiencia aórtica leve, y gradiente medio de $20 \mathrm{mmHg}$. De dos pacientes con estenosis supravalvular, uno quedó con estenosis residual importante. En cuanto a clase funcional, todos los pacientes se encuentran en grados 1 y 2 de la escala de NYHA. No se presentaron complicaciones inmediatas en los sometidos a procedimientos percutáneos o quirúrgicos. Fallecieron dos pacientes (10.5\%). Conclusiones: La estenosis aórtica afecta principalmente la región valvular, y el tratamiento tanto quirúrgico como percutáneo permiten aliviar la obstrucción hasta el momento de un reemplazo valvular.

Palabras claves: Estenosis aórtica, insuficiencia, valvuloplastia, bicúspide, dilatación, pediatría, resultados.

\begin{abstract}
Introduction: Aortic stenosis is relatively common in children. The objective of this study was to characterize the results and clinical course of this pathology after surgical and percutaneous management. Material and methods: This was a retrospective study of patients aged 0 to 19 years, treated for subvalvular, valvular, and supravalvular aortic stenosis, at Hospital de Clínicas, between 1998 and 2019. Cases associated with other congenital lesions were excluded. Results:Twenty patients were treated, age from 3 days to 17 years, male predominance $(80 \%)$. The stenosis was valvular in $65 \%$ of the cases, subvalvular $20 \%$, supravalvular $5 \%$ and multiple in $10 \% .61 \%$ of the valve recipients received percutaneous treatment, and the other surgical valvuloplasty. At follow-up, the free reoperation rate was $47 \%$ at 10 years; $50 \%$ of them have moderate to severe aortic valve regurgitation. Of the 13 cases of valve stenosis, treated percutaneously or surgically, 4 are awaiting valve replacement. Of 4 patients with subvalvular stenosis, 3 have mild aortic regurgitation and a mean gradient of 20 $\mathrm{mmHg}$. Of two patients with supravalvular stenosis, one was left with significant residual stenosis. Regarding functional class, all patients are in grades 1 and 2 of the NYHA scale. There were no immediate complications in those undergoing percutaneous or surgical procedures. Two patients $(10.5 \%)$ died. Conclusions: Aortic stenosis mainly affects the valve region, and both surgical and percutaneous treatment allow the obstruction to be relieved until the time of valve replacement.
\end{abstract}

Key words: Aortic stenosis, insufficiency, valvuloplasty, bicuspid, dilation, pediatrics, results.

\footnotetext{
${ }^{1}$ Universidad Nacional de Asunción. Facultad de Ciencias Médicas. Hospital de Clínicas. Departamento de Cardiología Pediátrica. San Lorenzo, Paraguay.

Correspondencia: Dong Chin Suh. Correo: dongchinsl@gmail.com

Conflicto de interés: Los autores declaran no poseer conflicto de interés.

Recibido: 25/03/2020 Aceptado:29/06/2020
}

Doi: https://doi.org/10.31698/ped.47022020008

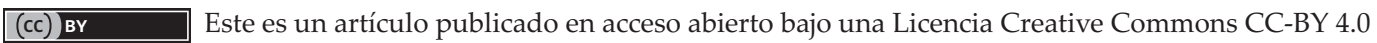




\section{INTRODUCCIÓN}

La incidencia de cardiopatías congénitas, es de aproximadamente 6 por 1.000 nacidos vivos, y la estenosis aórtica congénita representa aproximadamente el $5 \%$ de ellas ${ }^{(1,2)}$. Se caracteriza por el estrechamiento de la vía de salida del ventrículo izquierdo, ocasionando diferentes grados de obstrucción al pasaje de sangre del ventrículo izquierdo para la aorta, con un gradiente de presión durante la sístole y la consecuente sobrecarga de presión e hipertrofia ventricular. Puede afectar la válvula aórtica en $75 \%$ de los casos, la región subvalvular en $23 \%$ y la supravalvular en 1 a $2 \%$. Se asocia a otras patologías en un $20 \%$, como el ductus arterioso persistente, la coartación de aorta y el defecto del septo interventricular. Existen datos de que la estenosis aórtica es de etiología genética, y está asociada a algunos síndromes como el de Turner y el de Williams ${ }^{(3)}$.

Morfológicamente, en la estenosis valvular aórtica existe un engrosamiento y diversos grados de fusión comisural, siendo la válvula bicúspide en $64 \%$ de los casos. En la estenosis supravalvular, la obstrucción puede estar confinada a la unión sinotubular o ser difusa en la aorta ascendente. A su vez, la estenosis subvalvular, puede estar causada por una membrana fibromuscular o presentarse con un estrechamiento más difuso en la región subvalvular ${ }^{(4)}$.

La presentación clínica depende del grado de obstrucción, pudiendo manifestarse como un cuadro grave de insuficiencia cardiaca en la estenosis aórtica crítica del recién nacido. En pacientes mayores, el diagnóstico puede darse ante el hallazgo de un soplo, la disnea de esfuerzo, el dolor torácico o el síncope ${ }^{(5)}$.

Entre los exámenes complementarios, el electrocardiograma puede mostrar sobrecarga ventricular izquierda y alteraciones de la repolarización ventricular. El ecocardiograma suele ser suficiente en la mayoría de los casos como método de diagnóstico ${ }^{(5)}$.

El tratamiento es principalmente quirúrgico en los tipos supravalvular y subvalvular. La estenosis valvular puede ser tratada quirúrgicamente o por vía percutánea. Estas intervenciones sobre la vávula aórtica son consideradas paliativas, pues en la evolucion de la patología, el reemplazo valvular aórtico es prácticamente la norma ${ }^{(2)}$.

El objetivo de este trabajo fue describir los resultados y la evolución del manejo quirúrgico y percutáneo de pacientes pediátricos portadores de estenosis aórtica.

\section{MATERIALES Y MÉTODOS}

Estudio retrospectivo de tipo descriptivo, de casos pediátricos de estenosis aórtica, tratados y seguidos en el Hospital de Clínicas (FCM-UNA), durante el periodo de enero 1998 a marzo 2019. Fueron incluidos pacientes con diagnóstico de estenosis aórtica valvular, subvalvular y supravalvular; desde la edad neonatal hasta los 19 años. Los diagnósticos se dieron por la sospecha clínica, basada en el hallazgo de soplo cardiaco, síntomas de insuficiencia cardíaca, síncope y complicaciones infecciosas (endocarditis infecciosa), y posteriormente confirmados por ecocardiografía. Fueron excluidos los casos asociados a otras lesiones congénitas como comunicación interventricular o coartación aórtica. Se definió como tratamiento primario, al primer procedimiento quirúrgico o percutáneo realizado al paciente.

Como variables se describen los datos demográficos básicos (edad, sexo); la localización anatómica de la estenosis (subvalvular, valvular o supravalvular); en las valvulares se distinguió la presencia de una válvula aórtica bicúspide. Se buscó presencia de comorbilidades o síndromes genéticos. Se consideró como lesión mixta la presencia de afectación anatómica en más de una región del tracto de salida del ventrículo izquierdo. Otras variables fueron el tipo de procedimiento realizado: quirúrgico en las lesiones subvalvulares, supravalvulares y mixtas; y quirúrgico o percutáneo en el caso de las valvulares.

Se consignaron las complicaciones posprocedimiento y se cuantificó la reducción del gradiente ventrículo izquierdo-aorta por datos del cateterismo o la ecocardiografía, así como la aparición de insuficiencia 
aórtica. Se consideró como resultado adecuado la disminución del gradiente pico a pico de al menos $50 \%$ o un gradiente residual menor de $36 \mathrm{mmHg}$, con insuficiencia valvular trivial o leve. La cuantificación de la insuficiencia valvular aórtica se basó en los criterios angiográficos de Seller en el cateterismo y ecocardiográficamente según paramétros habituales (doppler espectral y doppler color).

Las variables continuas se expresan como media \pm desviación estándar y las variables categóricas fueron comparadas mediante el test de Fisher. El paquete estadístico utilizado fue MedCalc versión 17.9.5 (Ostende, Bélgica). El trabajo fue aprobado por la Dirección de investigaciones del hospital.

\section{RESULTADOS}

Fueron tratados de estenosis aórtica 20 pacientes. La edad al momento del primer procedimiento (quirúrgico o percutáneo) fue en promedio de $8.3 \pm$ 6.1 años. Hubo predominio del sexo masculino (80\%). La localización anatómica de la estenosis se detalla en la tabla 1.

Tabla 1. Localización anatómica de la obstrucción al tracto de salida del ventrículo izquierdo.

\begin{tabular}{lcc}
\hline Tipo de estenosis & número & Porcentaje \\
\hline Valvular & 13 & $65 \%$ \\
Subvalvular & 4 & $20 \%$ \\
Supravalvular & 1 & $5 \%$ \\
Valvular + supravalvular & 1 & $5 \%$ \\
Valvular + subvalvular & 1 & $5 \%$ \\
Total & $\mathbf{2 0}$ & $\mathbf{1 0 0} \%$ \\
\hline
\end{tabular}

Respecto a la presencia de comorbilidades, en un caso hubo asociación entre la estenosis supravalvular y el síndrome de Williams, y en otro la de estenosis valvular con toxoplasmosis congénita.

No se presentaron complicaciones en el postoperatorio de los sometidos a procedimientos percutáneos o quirúrgicos. Fallecieron dos pacientes (10.5\%) durante el periodo estudiado

\section{Estenosis valvular aórtica}

De 13 pacientes con estenosis valvular, en 8 de ellos el tratamiento primario fue la valvuloplastia percutánea con balón (edad promedio 8.2 años), y en los 5 restantes se realizaron como tratamiento primario: comisurotomía, valvulotomía transapical y recambio valvular. Ver tabla 2

La válvula aórtica bicúspide estaba presente en el $80 \%$ (12 de 15) de los casos de lesión valvular aislada o combinada. En el resto no hubo especificación de la morfología valvular.

Tabla 2. Procedimientos primarios en pacientes portadores de estenosis valvular aórtica (n: 13)

\begin{tabular}{lc}
\hline Tratamiento primario & Número \\
\hline Valvuloplastia percutánea & 8 \\
Valvulotomía transapical & 2 \\
Comisurotomía & 2 \\
Recambio valvular & 1 \\
\hline
\end{tabular}

Hubo dos casos de estenosis valvular aórtica crítica; el primero tenía diagnóstico prenatal y fue intervenido a los 3 días de vida; el segundo fue tratado al mes de vida; ambos recibieron infusión preoperatoria de prostaglandina E1 y la técnica utilizada fue la valvulotomía transapical quirúrgica.

En dos casos se realizó comisurotomía como primer tratamiento, debido a la morfología valvular mixomatosa en un paciente, y en el otro por lesión valvular secundaria a endocarditis bacteriana. El recambio valvular aórtico fue realizado como primer tratamiento en un caso con antecedente de endocarditis bacteriana reciente.

Por ecocardiografía, el gradiente sistólico promedio de la obstrucción a nivel valvular fue de $94.4 \pm 55$ $\mathrm{mmHg}$; en un caso, el gradiente fue de $22 \mathrm{mmHg}$, debido a una estenosis aórtica crítica asociada a disfunción ventricular. En los que se sometieron a valvuloplastia por balón, el gradiente pico a pico de ventrículo izquierdo-aorta fue de $114 \mathrm{mmHg}$ en promedio y el gradiente residual posvalvuloplastia percutánea fue de $52 \pm 33.4 \mathrm{mmHg}$.

Luego del procedimiento de dilatación con balón, el $50 \%$ de los pacientes quedó sin insuficiencia valvular o ésta era de grado leve. En la otra mitad, se constató progresión de la insuficiencia valvular aórtica de grados moderados a severos. 
En cuanto a la evolución de los 8 pacientes sometidos primariamente a dilatación por balón, tenemos que 5 de ellos se encuentran bajo seguimiento clínico, con un gradiente promedio de $45 \mathrm{mmHg}$ por ecocardiografía. De los 3 restantes, 2 pacientes se encuentran aguardando tratamiento de reemplazo valvular debido a una insuficiencia valvular severa en un caso, y en el otro por estenosis valvular residual severa asociada a seudoaneurisma del seno coronario. El tercer paciente ya recibió el reemplazo valvular luego de dos procedimientos de dilatación percutánea previos. Ver tabla 3.

Tabla 3. Evolución de pacientes sometidos a valvuloplastia con balón. N: 7

\begin{tabular}{lcccc}
\hline Nombre y edad actual & Gradiente residual & Insuficiencia aórtica & Morfología valvular & Planes \\
\hline F.F. 19 años & 42 & no & bicúspide & Seguimiento clínico \\
J.G. 7 años & 36 & leve & bicúspide & Seguimiento clínico \\
C.G. 20 años & 34 & moderada & bicúspide & Seguimiento clínico \\
R.E. 17 años & 37 & moderada & bicúspide & Seguimiento clínico \\
M.L. 1 año & 78 & moderada & bicúspide & Seguimiento clínico \\
N.B. 12 años & 118 & leve & bicúspide & RV \\
G.M. 18 años & 63 & severa & bicúspide & RV \\
\hline
\end{tabular}

Gradiente residual: en $\mathrm{mmHg}$ (ecocardiografía) RV: reemplazo valvular

\section{Estenosis subvalvular y supravalvular}

Los casos de estenosis aórtica subvalvular y supravalvular fueron tratados quirúrgicamente mediante miectomía o aortoplastia con parche de Dacrón respectivamente. Las estenosis valvulares asociadas a lesión supra o subvalvar fueron tratadas quirúrgicamente en un solo tiempo. Por ecocardiografía, el gradiente sistólico promedio de la obstrucción a nivel infravalvular fue de $81 \mathrm{mmHg}$ y en las lesiones supravalvulares de $69 \mathrm{mmHg}$.

\section{Seguimiento}

El seguimiento fue de 6.3 años en promedio (rango: 1 a 19 años). Todos los pacientes se encuentran asintomáticos, y en los casos con lesiones residuales significativas, se ha indicado la restricción de actividades físicas extenuantes.

Considerando todos los pacientes, el periodo libre de reintervención a los 5 años fue del $64,6 \%$ en los sometidos a tratamiento quirúrgico y $71,4 \%$ para el tratamiento percutáneo; con un periodo libre de reintervención a los 10 años de 43,1\% y 47,6\% respectivamente.

En el grupo de pacientes que se encuentran estables respecto a su lesión (11 casos, 52.6\%), el gradiente sistólico fue de $27.3 \pm 14.8 \mathrm{mmHg}$ y en los 7 pacientes restantes en quienes se plantea una reintervención el gradiente sistólico fue de $90.2 \pm 24.5 \mathrm{mmHg}$.

De 8 pacientes con estenosis valvular aórtica tratados primariamente con balón, 2 están en planes de reintervención quirúrgica; uno por estenosis severa y aneurisma del seno coronario, y el otro paciente, por estenosis moderada e insuficiencia severa. El tiempo de seguimiento promedio de los pacientes tratados percutáneamente fue de 7.1 años (rango 4 a 19 años).

De los 4 pacientes con estenosis valvular tratados quirúrgicamente en forma primaria, 3 se encuentran aguardando reintervención por estenosis severa residual; con un periodo promedio de seguimiento de 8.5 años (rango 2 a 19 años). En la tabla 4 se detallan los pacientes con necesidad de reintervención. 
Tabla 4. Pacientes en planes de reintervención n: 6

\begin{tabular}{|c|c|c|c|c|c|c|c|}
\hline Paciente & $\begin{array}{l}\text { Edad actual } \\
\text { (años) }\end{array}$ & Diagnóstico & $\begin{array}{l}\text { Tratamiento } \\
\text { realizado }\end{array}$ & $\begin{array}{l}\text { Tiempo seguimiento } \\
\text { (años) }\end{array}$ & Lesión residual & ECG & $\begin{array}{c}\text { Tratamiento } \\
\text { propuesto }\end{array}$ \\
\hline R. V. & 12 & ESpV & Plastia quirúrgica & 5 & $\begin{array}{l}\text { Estenosis supravalvular } \\
\text { Grad: } 130\end{array}$ & SVI & $\begin{array}{c}\text { Plastia } \\
\text { quirúrgica }\end{array}$ \\
\hline C. D. & 18 & EV & Plastia quirúrgica & 5 & $\begin{array}{l}\text { Estenosis valvular Grad; } 90 \\
\text { Insuficiencia aórtica moderada }\end{array}$ & ARV & $\begin{array}{l}\text { Recambio } \\
\text { valvular }\end{array}$ \\
\hline G. M. & 18 & EV & Dilatación percutánea & 6 & $\begin{array}{l}\text { Insuficiencia aórtica severa } \\
\text { Estenosis residual Grad: } 63\end{array}$ & ARV & $\begin{array}{l}\text { Recambio } \\
\text { valvular }\end{array}$ \\
\hline N. B. & 12 & EV & Dilatación percutánea & 4 & $\begin{array}{l}\text { Estenosis valvular. Grad: } 118 \\
\text { Aneurisma del seno coronario }\end{array}$ & $S V I+A R V$ & $\begin{array}{l}\text { Recambio } \\
\text { valvular }\end{array}$ \\
\hline V.I. & 12 & EV & Recambio valvular & 8 & Estenosis valvular Grad; 75 & SVI sin ARV & $\begin{array}{l}\text { Recambio } \\
\text { valvular }\end{array}$ \\
\hline J. R. & 3 & EV & Valvutomía transapical & 3 & $\begin{array}{l}\text { Estenosis valvular Grad: } 78 \\
\text { Insuficiencia aórtica leve-mod }\end{array}$ & ARV & $\begin{array}{l}\text { Dilatación } \\
\text { percutánea }\end{array}$ \\
\hline
\end{tabular}

ESpV: estenosis supravalvular Grad: gradiente en $\mathrm{mmHg}$ SVI: sobrecarga de ventrículo izquierdo ARV: alteraciones de repolarización ND: no disponible

Respecto a los 4 pacientes operados de estenosis subvalvular, 3 de ellos se encuentran con insuficiencia aórtica leve, y gradiente promedio de 20 mmHg. Un paciente, con insuficiencia aórtica moderada, presentó gradiente residual de $46 \mathrm{mmHg}$.

De los dos pacientes tratados estenosis supravalvular, uno se encuentra con estenosis residual importante, estando en planes de reoperación.

En cuanto a clase funcional, todos los pacientes se encontraban en los grados 1 y 2 de la escala de Ross o NYHA. A los portadores de estenosis residual moderada se les restringe la realización de deportes. El electrocardiograma más reciente estuvo disponible en 13 pacientes. En cuatro de ellos, sin lesiones residuales importantes, el trazado era totalmente normal. Los hallazgos principales en los pacientes con planes de reintervención fueron la sobrecarga ventricular izquierda y las alteraciones de la repolarización ventricular (anomalías de la onda $\mathrm{T}$ ).

Respecto a la insuficiencia valvular aórtica, en los intervenidos percutáneamente por estenosis valvular, ha sido progresiva en el seguimiento, presentándose en el 50\% de ellos (4/8), de grado moderado a severo. En las estenosis valvulares manejadas quirúrgicamente, el $75 \%$ de los pacientes (3/4) quedó con insuficiencia de grado moderado. En las lesiones subaórticas, solo 1 de 4 pacientes, evolucionó a insuficiencia moderada, el resto se mantiene sin insuficiencia o ésta es mínima. De los dos pacientes con lesiones supravalvulares, uno tiene insuficiencia aórtica leve.

\section{DISCUSIÓN}

La estenosis aórtica es una patología relativamente frecuente en la edad pediátrica, cuyas complicaciones e implicancias se extienden hasta la edad adulta. En esta serie encontramos pacientes tratados ya desde edad neonatal, con un predominio del sexo masculino. El tratamiento quirúrgico de las lesiones subvalvulares y supravalvulares en general, permite la curación de la patología. Sin embargo, en las lesiones valvulares los tratamientos iniciales, tanto los quirúrgicos o percutáneos, permiten aliviar la obstrucción, permitiendo disminuir el riesgo de muerte súbita y arritmias, hasta que según la evolución, el paciente requiera del recambio de la válvula aórtica por una prótesis ${ }^{(4)}$. Como menciona la literatura, la estenosis valvular aórtica es la principal lesión obstructiva del tracto de salida del ventrículo izquierdo; en esta revisión representó el 65\% de los casos. Su etiología congénita involucra a la válvula aórtica bicúspide en su mayor porcentaje. Las indicaciones de tratamiento se basan en la presencia de síntomas y en el grado de obstrucción; se consideran gradientes significativos los valores mayores a $50 \mathrm{mmHg}$ en el cateterismo y mayores a 60-70 mmHg en el ecocardiograma. La presencia de alteraciones del segmento ST y la onda T en el electrocardiograma en reposo o con el esfuerzo también son factores a considerar en el manejo. El abordaje terapéutico de la estenosis valvular aórtica 
depende de varios factores, como la edad, el grado de obstrucción, la morfología valvular, la condición clínica del paciente y la experiencia y posibilidades del equipo médico tratante en cuanto a un manejo quirúrgico o percutáneo $^{(6)}$.

\section{Estenosis valvular aórtica}

En las lesiones valvulares, la dilatación percutánea con balón es una de las estrategias más utilizadas, considerando siempre en su elección, las variables anatómicas y los recursos institucionales. En nuestra serie, hemos encontrado que el $75 \%$ de los tratados percutáneamente, se mantenían sin necesidad de reintervención en un promedio de 7 años de seguimiento. En el grupo tratado quirúrgicamente, el 75\% se encuentra en planes de recambio valvular aórtico (seguimiento promedio de 8.5 años). Aunque en una revisión de Donald et al., comparando la valvuloplastia quirúrgica con la dilatación percutánea en la estenosis valvular aórtica, se demostró un mayor porcentaje de pacientes libres de reoperación a los 10 años con la primera estrategia, y con mortalidad temprana similares, sigue siendo la valvuloplastia percutánea la primera opción, principalmente en niños mayores ${ }^{(7)}$.

El tratamiento quirúrgico primario de las lesiones valvulares (comisurotomía o reemplazo valvular), se dio principalmente en pacientes con endocarditis infecciosa concomitante o en los casos de lesiones de múltiple localización.

La valvulotomía transapical quirúrgica, históricamente, es una de las técnicas utilizadas desde antes de la introducción de la valvuloplastia con balón, y ofrece resultados aceptables en los casos de estenosis aórtica crítica en recién nacidos. La decisión sobre una u otra técnica, depende de una evaluación de riesgo, el estado clínico del paciente y la disponibilidad. Uno de nuestros pacientes sometidos a valvulotomía transapical en etapa neonatal, recibió reemplazo valvular aórtico a los 19 años de edad ${ }^{(8)}$.

En esta revisión se constató una frecuencia de insuficiencia aórtica como complicación secundaria a la dilatación percutánea del $25 \%$. La insuficiencia valvular aórtica, puede estar presente antes del tratamiento, aparecer como complicación de él, o progresar independientemente. Por ello, la comparación entre las diferentes técnicas respecto a la prevalencia de insuficiencia valvular aórtica es problemática, debido a que su evolución es dependiente de factores anatómicos, dados principalmente por la morfología valvular bicúspide. En una revisión de más de mil pacientes sometidos a dilatación por balón, en el seguimiento constataron cifras de $34 \%$ de insuficiencia valvular aórtica de grado mayor a leve $\mathrm{e}^{(9)}$.

La mortalidad operatoria se dio en un paciente con lesión subaórtica y valvular, que tuvo que ser reintervenido a los pocos días debido a persistencia de la obstrucción subaórtica. El otro paciente fallecido, estaba en planes de recambio valvular aórtico por recurrencia de la estenosis valvular (tenía gradiente de $130 \mathrm{mmHg}$ en el ecocardiograma y alteraciones de la repolarización ventricular); presentó muerte súbita luego de realizar deportes. La mortalidad operatoria temprana en la revisión de Brown et al. fue de $11 \%$.

Respecto a la utilización de técnicas más antiguas como la dilatación valvular por vía transpical, podemos mencionar que factores inherentes al paciente (estado clínico, peso) e institucionales (experiencia, disponibilidad de hemodinamistas) son los que determinan su uso. En la actualidad, la dilatación percutánea ha reemplazado a los demás abordajes quirúrgicos ${ }^{(8)}$.

\section{Estenosis subvalvular}

Al considerar las lesiones subvalvulares se presentan varios aspectos. La necesidad de aliviar la obstrucción para disminuir el riesgo de muerte súbita, la posibilidad de recidiva, y la insuficiencia valvular progresiva que puede ser independiente de la corrección del defecto. Todo esto implica el seguimiento a largo plazo de estos pacientes, ya que la cirugía no es curativa. En nuestra revisión, hemos encontrado en un caso el empeoramiento de la insuficiencia aórtica, coincidente con la persistencia de una estenosis subaórtica moderada ${ }^{(4)}$.

\section{Estenosis supravalvular}

En general, el pronóstico a largo plazo de la corrección de la estenosis supravalvular es bueno. Se 
describen casos de persistencia de la obstrucción y de desarrollo de insuficiencia valvular aórtica por presencia de tejidos fibrosos residuales en la íntima ${ }^{(10)}$. Hemos encontrado ambas situaciones en los dos casos de estenosis supravalvular; en un caso, estenosis residual importante y en el otro, insuficiencia valvular aórtica leve.

Como limitaciones, debemos considerar, las inherentes a un trabajo retrospectivo y el pequeño número de pacientes.

\section{CONCLUSIONES}

La presentación clínica de la estenosis aórtica es variable, desde un estado de choque cardiogénico en recién nacidos, pacientes asintomáticos con hallazgo de soplo y hasta casos de muerte súbita.

\section{REFERENCIAS}

1. Hoffman JI. Incidence of congenital heart disease: I. Postnatal incidence. Pediatr Cardiol 1995;16:103-113. doi: http://dx.doi.org/10.1007/BF00801907

2. Brown JW, Ruzmetov M, Vijay P, Rodefeld MD, Turrentine MW. Surgery for aortic stenosis in children: a 40-year experience. Ann Thorac Surg. 2003; 76: 1398-1411. doi: https://doi.org/10.1016/S0003-4975(03)01027-0

3. Brown JW, Stevens LS, Holly S, Robinson R, Rodefeld M, Grayson T, et al. Surgical spectrum of aortic stenosis in children: a thirty-year experience with 257 children. Ann Thorac Surg. 1988;45(4):393-403. doi: https://doi.org/10.10 16/s0003-4975(98)90012-1

4. Abella R, Fernández-Doblas J, Ferrer Q, Gran F. Estenosis aórtica. En: Díaz GF, Sandoval N, Vélez JF, editores. Cardiología Pediátrica. 2ํㅡㄹ edición. Bogotá: Distribuna Editorial; 2017.p. 841-865.

5. Haas NA, Schirmer KR. Guidelines for the Management of Congenital Heart Diseases in Childhood and Adolescence. Cardiol Young. 2017;27(S3):S1-S105. doi: https://doi.org/10.1017/S1047951116001955

6. Alva C, Gómez FD, Yáñez L. Estenosis valvuar aórtica. Actualización del tratamiento. Arch Cardiol Mex. 2006;76(S4):152-157.
El tratamiento es prácticamente paliativo en las estenosis valvulares, ya que en un tiempo variable, finalmente todos los pacientes llegarán a un recambio valvular protésico. La recurrencia de la estenosis y el progresivo empeoramiento de la insuficiencia valvular, es una complicación posible en las tres variedades anatómicas (subvalvular, valvular y supravalvular).

El tratamiento percutáneo es el tratamiento de elección en la mayoría de las situaciones clínicas en la estenosis valvular aórtica. Este procedimiento tiene la ventaja de reducir el grado de obstrucción, disminuyendo el riesgo de muerte súbita y es menos invasivo al no precisar de esternotomía y circulación extracorpórea.

7. Donald JS, Konstantinov IE. Surgical aortic valvuloplasty versus balloon aortic valve dilatation in children. World J Pediatr Congenit Heart Surg. 2016;7(5):583-591. doi: https://doi.org/10.1177/2150135 116651091

8. Brown JW, Ruzmetov M, Vijay P, Rodefeld MD, Turrentine MW. Closed transventricular aortic valvotomy for critical aortic stenosis in neonates: outcomes, risk factors, and reoperations. Ann Thorac Surg. 2006;81(1):23642. doi: https://doi.org/10.1016/j.athoracsur.2005.06.075

9. Ewert P, Bertram H, Breuer J, Dähnert I, Dittrich S, Eicken A, et al. Balloon valvuloplasty in the treatment of congenital aortic valve stenosis-a retrospective multicenter survey of more than 1000 patients. Int J Cardiol. 2011;149(2):182-185. doi: https://doi.org/10.1016/j.ijcard.2 010.01 .005

10. Brown JW, Ruzmetov M, Vijay P, Turrentine MW. Surgical repair of congenital supravalvular aortic stenosis in children. Eur J Cardiothorac Surg. 2002;21(1):50-6. doi: https://doi.org/10.1016/s1010-7940(01)01087-9 